\title{
New Suns in the Cosmos II: differential rotation in Kepler Sun-like stars
}

\author{
M. L. Das Chagas, ${ }^{1,2}$ J. P. Bravo, ${ }^{1}$ A. D. Costa, ${ }^{1}$ C. E. Ferreira Lopes, ${ }^{3}$ \\ R. Silva Sobrinho, ${ }^{1}$ F. Paz-Chinchón, ${ }^{4,5}$ I. C. Leão,${ }^{6}$ A. Valio, ${ }^{7}$ D. B. de Freitas, ${ }^{1}$ \\ B. L. Canto Martins, ${ }^{1}$ A. F. Lanza ${ }^{8}$ and J. R. De Medeiros ${ }^{1 \star}$ \\ ${ }^{1}$ Departamento de Física Teórica e Experimental, Universidade Federal do Rio Grande do Norte, Natal, RN 59078-970, Brazil \\ ${ }^{2}$ Faculdade de Física - Instituto de Ciências Exatas, Universidade Federal do Sul e Sudeste do Pará, Marabá, PA 68505-080, Brazil \\ ${ }^{3}$ SUPA Wide-Field Astronomy Unit, Institute for Astronomy, School of Physics and Astronomy, University of Edinburgh, \\ Royal Observatory, Blackford Hill, Edinburgh EH9 3HJ, UK \\ ${ }^{4}$ Departamento de Enseñanza de las Ciencias Básicas, Universidad Católica del Norte, Larrondo 1281, Coquimbo 17814-21, Chile \\ ${ }^{5}$ Millennium Institute of Astrophysics, Av. Vicuña Mackenna 4860, Macul, Santiago 78204-36, Chile \\ ${ }^{6}$ European Southern Observatory, Karl-Schwarzschild-Strasse 2, Garching D-85748, Germany \\ ${ }^{7}$ CRAAM, Universidade Presbiteriana Mackenzie, Rua da Consolação 896, São Paulo, SP 01302, Brazil \\ ${ }^{8}$ INAF, Osservatorio Astrofisico di Catania, via S. Sofia 78, Catania I-95123, Italy
}

Accepted 2016 August 10. Received 2016 August 8; in original form 2016 April 5

\begin{abstract}
The present study reports the discovery of Sun-like stars, namely main-sequence stars with $T_{\text {eff }}, \log g$ and rotation periods $P_{\text {rot }}$ similar to solar values, presenting evidence of surface differential rotation (DR). An autocorrelation of the time series was used to select stars presenting photometric signal stability from a sample of 881 stars with light curves collected by the Kepler space-borne telescope, in which we have identified 17 stars with stable signals. A simple two-spot model together with a Bayesian information criterion were applied to these stars in the search for indications of DR; in addition, for all 17 stars, it was possible to compute the spot rotation period $P$, the mean values of the individual spot rotation periods and their respective colatitudes, and the relative amplitude of the DR.
\end{abstract}

Key words: stars: rotation-stars: solar-type-starspots.

\section{INTRODUCTION}

Stars are normally born with rapid rotation, and the angular velocity distribution will be established in their infancy stages, mostly as the result of the interaction of the stellar magnetic field with the circumstellar accretion disc, at least for late-type stars (e.g. Shu et al. 1994; Bouvier, Forestini \& Allain 1997; van Saders \& Pinsonneault 2013). During the initial stages, the surfaces of stars with convective envelopes will slow down via magnetic braking resulting from the interaction between the stellar magnetic field and the magnetized wind from the surface (e.g. Kawaler 1988; Reiners \& Mohanty 2012).

Surface rotation can now be measured for many families of stars using different procedures, including the analyses of spectral line broadening, which produces projected rotational velocity $v \sin (i)$ measurements (e.g. De Medeiros \& Udry 1999; Nordström et al. 2004; De Medeiros et al. 2014), and periodic modulation of starlight produced by non-uniformities on the surface of the stars (e.g. Affer et al. 2012; De Medeiros et al. 2013; McQuillan, Mazeh \& Aigrain 2014; Leão et al. 2015). Other procedures include those based on

^E-mail: renan@dfte.ufrn.br line core variations in the $\mathrm{Ca}$ II $\mathrm{H}$ and $\mathrm{K}$ lines (e.g. Baliunas et al. 1983) and on the Rossiter-McLaughlin effect or ellipsoidal light variations in eclipsing binaries.

In addition, it is now well established that the surface and internal stellar rotation pattern is by no means uniform. For instance, Helioseismology has revealed a large spread of rotation rates in the outer convective regions at different latitudes, with the inner regions presenting an almost constant rotation rate (e.g. Aerts et al. 2010). These aspects are intimately associated with the stellar differential rotation (hereafter DR), i.e. the property that different parts of the star rotate at different rates (Miesch 2005; Miesch \& Toomre 2009; Kitchatinov 2013). The current leading theoretical basis, first presented by Lebedinsky (1941), explains DR based on the interaction between convection and rotation, with convective motions in a rotating star being disturbed by the Coriolis force. Its back reaction redistributes angular momentum and disturbs the global rotation behaviour to produce non-uniformities, leading to DR of the surface.

Different procedures can be used in the diagnosis of surface DR. In the first procedure, Doppler imaging, the positions of individual spots are estimated based on their effects on the stellar spectral line profiles, and on the condition that the star is rotating rapidly enough (e.g. Collier Cameron, Donati \& Semel 2002). In the second 
procedure, the Fourier transform method, the Doppler shift at different latitudes due to rotation can be estimated from the Fourier transform of the line profiles (e.g. Reiners \& Schmitt 2003; Reinhold $\&$ Reiners 2013). In the third procedure, time series photometry, the rotation periods can be computed from a time series of photometric observations (e.g. Lanza, Das Chagas \& De Medeiros 2014; Aigrain et al. 2015; Davenport, Hebb \& Hawley 2015). Another approach is based on asteroseismology, in which the frequency splitting of global oscillations is explained in terms of different latitudinal rotation rates (e.g. Gizon \& Solanki 2004). A recent blind survey of competing techniques for detecting rotation and DR from model photometry, conducted by Aigrain et al. (2015), showed excellent agreement in recovering the overall rotation periods for stars exhibiting low and moderate activity levels. However, the referred study revealed a complex degeneracy between DR shear, spot lifetimes and the number of spots present, suggesting that DR studies based on full-disc light curves alone need to be treated with caution.

The advent of the space-borne CoRoT (Baglin et al. 2006) and Kepler (Borucki et al. 2010) telescopes made it possible to study in great detail the behaviour of the rotation of Sun-like stars. In this context, a large effort is being directed at the analysis of more active stars using the photometric modulations observed from their light curve (e.g. Bonomo \& Lanza 2012; Fröhlich et al. 2012; De Medeiros et al. 2013; McQuillan, Aigrain \& Mazeh 2013), therein producing rotation periods for thousands of different families of stars. A parallel effort is being made by different authors to enlarge the horizons of our quantitative and qualitative understanding of DR (e.g. Reinhold \& Reiners 2013; Reinhold, Reiners \& Basri 2013; Lanza et al. 2014; Aigrain et al. 2015; Reinhold \& Gizon 2015).

Most of the DR surface patterns observed to date are predominantly solar type, with rotation rates decreasing from the equatorial to polar regions (e.g. Baliunas et al. 1983; Lanza, Rodono \& Zappala 1993; Collier Cameron et al. 2002; Reiners \& Schmitt 2003; Reinhold \& Reiners 2013; Lanza et al. 2014). The DR total surface gradient varies to a high degree with the effective temperature (Barnes et al. 2005) and to a low degree with the rotation rate (Küker \& Rüdiger 2005). Antisolar DR measurements are sparse and have mainly been performed for some late-type giant stars, most of which being components of RS Canum Venaticorumsystems (e.g. Strassmeier, Kratzwald \& Weber 2003; Oláh, Jurcsik \& Strassmeier 2003; Weber, Strassmeier \& Washuettl 2005; Vida et al. 2007). As noted by different authors (e.g. Kovári et al. 2015), it appears that the strength and even the orientation of the DR are influenced by close companions, although such a scenario is not yet understood.

By applying asteroseismology procedures to time series obtained from light curve (hereafter LC) data from the Kepler or CoRoT missions, we are now in a position to extract, in addition to information about the surface rotational pattern, the physical characteristics of the stellar interior, revealing not only relevant aspects of DR but also information about pulsation modes and important constraints for dynamo models of low-mass stars. This enables one to test theoretical models for internal DR (see, e.g. Kitchatinov \& Olemskoy 2011; Küker \& Rüdiger 2011a), as well as the development of 3D simulations (e.g. Brun 2004; Browning 2008; Käpylä, Mantere \& Brandenburg 2012). Further, it has been possible to estimate the ratio between the rotation rate in the small helium core and the large convective regions of late-type stars (e.g. Eggenberger et al. 2010).

This is the second paper of a series of studies devoted to the identification of Sun-like stars presenting physical properties similar to the Sun. In the first study (De Freitas et al. 2013), we identified stars representing potentially good matches to the Sun's rotation.

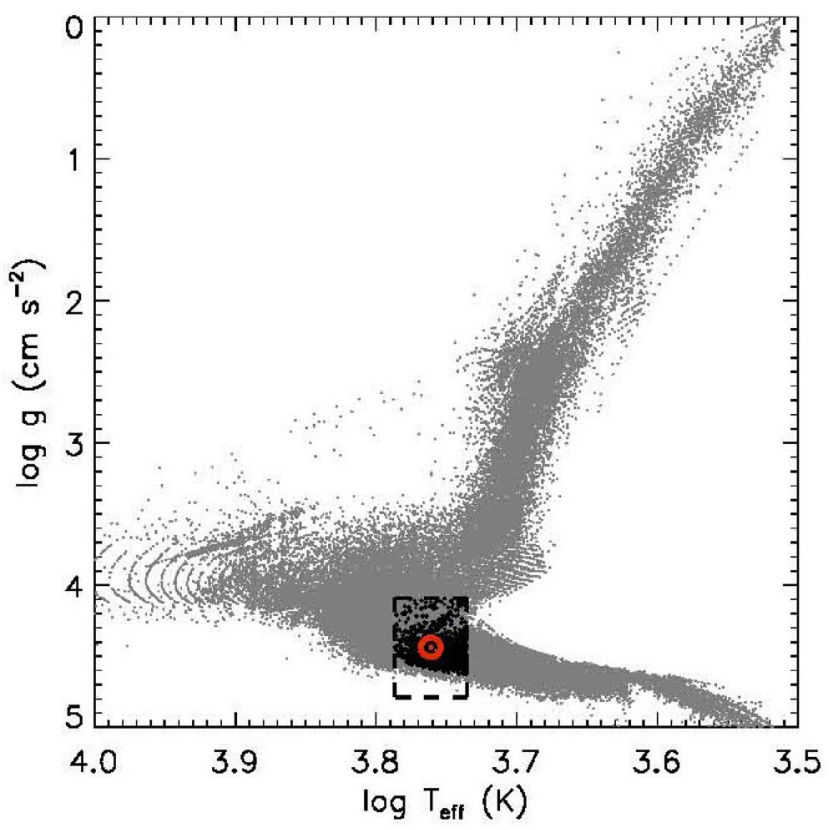

Figure 1. Distribution of $\log g$ and $T_{\text {eff }}$ from entire Kepler data base. The black rectangle denotes the region of sources with solar parameters with $T_{\text {eff }}^{\odot}$ and $\log g \odot$. The red circle shows the position of the Sun, and the small black cross the stars of the sample analyzed.

The main goal of this work is to apply spot modelling (Lanza et al. 2014) for a large sample of Sun-like stars observed in the scope of the Kepler mission, therein attempting to measure DR and quantify how common DR is among Sun-like stars presenting solar parameters and, in particular, stars with similar Sun rotation periods. This paper is organized as follows: Section 2 presents the stellar sample with the Kepler stellar parameters. In Section 3, we introduce the autocorrelation function (ACF). Results and Conclusions are presented in Sections 4 and 5, respectively.

\section{WORKING SAMPLE AND DATA ANALYSES}

From 2009 May to 2013 May, the Kepler mission collected data in a steady field of view for 191449 stars in 17 runs (known as quarters), which were composed of long-cadence (6.02 s observations stacked every 29.4 min; Jenkins et al. 2010) and short-cadence (bins of $59 \mathrm{~s}$ ) observations (Van Cleve et al. 2010; Thompson et al. 2013). For this study, we selected the calibrated LCs processed by the PDC_MAP pipeline (Jenkins et al. 2010). To search for stars with physical properties approximately equal to the solar values, we made an initial selection of LCs from the Kepler data base (Mikulski Archive for Space Telescopes $\left.{ }^{1}\right)$ using the solar parameters $\log g(\sim 4.44) \mathrm{cm} \mathrm{s}^{-2}$, $T_{\text {eff }}(\sim 5779) \mathrm{K},[\mathrm{Fe} / \mathrm{H}](\sim 0$. $)$ dex and $23 \mathrm{~d}<P_{\text {rot }}<33 \mathrm{~d}$. A total of 881 stars with $3.94 \mathrm{~cm} \mathrm{~s}^{-2}<\log g<4.94 \mathrm{~cm} \mathrm{~s}^{-2}, 5579 \mathrm{~K}$ $<T_{\text {eff }}<5979 \mathrm{~K}$, were selected, with effective temperature and gravity obtained from Huber et al. (2014) and rotation period given by McQuillan et al. (2013). The location of our working sample, in the $\log g$ versus $T_{\text {eff }}$ diagram, in the context of the entire Kepler stellar sample, is displayed in Fig. 1. With such a working sample at hand, a careful treatment was applied to the LCs, using the so-called co-trending basis vectors provided by the Kepler archive (see Twicken et al. 2010; Smith et al. 2012; Stumpe et al. 2012), to

\footnotetext{
${ }^{1}$ http://archive.stsci.edu/kepler/data_search/search.php, hereafter MAST
} 
Table 1. Initial and final times of the intervals considered for the MCMC analysis, together with the BIC-computed values.

\begin{tabular}{lccc}
\hline $\begin{array}{l}\text { Star } \\
(\text { KIC \#) }\end{array}$ & $\begin{array}{c}t_{1} \\
(\text { BKJD })\end{array}$ & $\begin{array}{c}t_{2} \\
(B K J D)\end{array}$ & BIC \\
\hline 2831979 & 1212.163 & 1255.382 & 4.097 \\
4820062 & 735.384 & 761.804 & 18.778 \\
5781991 & 131.513 & 164.984 & 5.300 \\
5956717 & 1182.758 & 1207.585 & 8.027 \\
6143158 & 411.224 & 439.158 & 17.008 \\
6836955 & 261.205 & 293.591 & 10.65 \\
7430659 & 863.035 & 899.592 & 3.261 \\
8024188 & 1201.311 & 1240.893 & 7.467 \\
8037792 & 634.978 & 676.926 & 16.325 \\
8495770 & 844.746 & 880.383 & 19.635 \\
9996105 & 264.290 & 336.132 & 3.183 \\
10079452 & 448.517 & 499.623 & 8.413 \\
10279927 & 1216.781 & 1239.504 & 18.279 \\
10460082 & 416.803 & 442.203 & 11.926 \\
10514649 & 205.096 & 247.352 & 4.528 \\
11199277 & 1419.912 & 1465.927 & 14.378 \\
12520213 & 820.143 & 869.328 & 7.102 \\
\hline
\end{tabular}

remove systematic long-term trends originating from instruments, the environment, the detector or effects caused by the re-orientation of the spacecraft after each $\sim 90 \mathrm{~d}$. To remove outliers and prepare the LCs for the analysis using spot modelling, we applied the method developed by De Medeiros et al. (2013), a procedure that is able to identify discontinuities in the LCs, similar to that used by Basri et al. (2011). From this point on, an LC was considered to be fully treated, and its spot-modelling analysis could be performed.

\section{THE AUTOCORRELATION METHOD}

In this work, we follow the same procedure developed by Lanza et al. (2014) to estimate surface DR. First, we applied an ACF to check the stability of the photometric signal. Indeed, an important feature of the ACF is that it exhibits an oscillatory behaviour with regularly spaced peaks; then, the coherence of a photometric signal can be estimated by the relative height of successive peaks in the ACF (Lanza et al. 2014). A crucial step in our analysis was the search for photometric signal stability for all 881 LCs constituting our initial working sample. From such analyses, we identified 17 stars presenting unambiguous stable photometric signals, indicating rotational modulation. Nevertheless, in spite of the fact that a significant DR can be detected when the relative height of the second maximum in the ACF is at least 0.6-0.7 (Lanza et al. 2014), we have considered a few stars whose ACF has a peak ratio smaller than this threshold because the Monte Carlo Markov chain (MCMC) analysis points for a significant DR for them. These stars with less good ACFs are flagged by a dagger in Table 2 . Indeed, the ACF has been widely used in the study of photometric signals due to its ability to provide a good estimate of the average period variability, including stellar rotation period (e.g. Affer et al. 2012; McQuillan et al. 2013). Then, for these 17 stars with sufficiently stable signals, we applied spot modelling (Lanza et al. 2014) to seek individual spot rotation periods. The method of spot modelling is based on two spots and was applied with a Bayesian information criterion (hereafter BIC) to initially choose intervals of the time series presenting evidence of DR with starspots of almost constant areas. The initial and final times $t_{1}$ and $t_{2}$, respectively, of those intervals are given in Table 1 , together with the BIC-computed values for each of the 17 stars. Indeed, $t_{1}$ and $t_{2}$ are defined in Barycentric Kepler Julian Day (BKJD). Even if the time intervals are particularly small, the spot modelling is able to give us a valid signal of DR, as many other authors (e.g. Croll et al. 2006; Fröhlich 2007) have proven in previous studies. Readers are referred to Lanza et al. (2014) for a complete discussion of the ACF and the spot-modelling procedure. Nevertheless, let us underline an important aspect, previously considered by different authors (e.g. Jeffers \& Keller 2009; Davenport et al. 2015), in the context of the present procedure. In the applied two-spot modelling, we cannot constrain the total number of starspots on the stellar surface, which, as noted by Davenport et al. (2015), may reflect two groups of spots or even many small spots across the entire stellar surface.

The LCs and the oscillatory behaviour of the ACF for these 17 stars are shown in Fig. A1 of Appendix. The blue vertical solid

Table 2. The stellar parameters and the results of MCMC analysis for our sample of 17 stars with traces of DR.

\begin{tabular}{|c|c|c|c|c|c|c|c|c|c|c|}
\hline $\begin{array}{l}\text { Star } \\
(\text { KIC \#) }\end{array}$ & $\begin{array}{l}P_{\text {rot }} \\
\text { (d) }\end{array}$ & $\begin{array}{c}\log g \\
\left(\mathrm{~cm} \mathrm{~s}^{-2}\right)\end{array}$ & $\begin{array}{l}T_{\text {eff }} \\
(\mathrm{K})\end{array}$ & $\begin{array}{l}P_{1} \\
\text { (d) }\end{array}$ & $\begin{array}{c}\sigma P_{1} \\
\text { (d) }\end{array}$ & $\begin{array}{l}P_{2} \\
\text { (d) }\end{array}$ & $\begin{array}{l}\sigma P_{2} \\
\text { (d) }\end{array}$ & $\Delta P / P$ & $\sigma \Delta P / P$ & $\begin{array}{l}\Delta \Omega \\
\text { (d) }\end{array}$ \\
\hline 2831979* & 24.383 & 4.363 & 5783 & 24.433 & $9.597 \times 10^{-4}$ & 22.660 & $1.629 \times 10^{-3}$ & 0.0753 & $8.332 \times 10^{-5}$ & 0.02012 \\
\hline $5781991^{\dagger}$ & 31.464 & 4.466 & 5796 & 28.947 & $3.150 \times 10^{-2}$ & 33.006 & $4.909 \times 10^{-2}$ & 0.1310 & $2.007 \times 10^{-3}$ & 0.02669 \\
\hline $5956717^{*}$ & 23.297 & 4.213 & 5657 & 24.069 & $9.727 \times 10^{-4}$ & 21.828 & $6.862 \times 10^{-4}$ & 0.0976 & $5.440 \times 10^{-5}$ & 0.02679 \\
\hline $6143158^{\dagger}$ & 23.155 & 4.509 & 5696 & 25.513 & $6.648 \times 10^{-3}$ & 21.448 & $2.218 \times 10^{-3}$ & 0.1731 & $3.243 \times 10^{-4}$ & 0.04667 \\
\hline $6836955^{\dagger}$ & 26.354 & 4.61 & 5590 & 24.000 & $9.132 \times 10^{-3}$ & 26.724 & $5.223 \times 10^{-3}$ & 0.1074 & $4.371 \times 10^{-4}$ & 0.02668 \\
\hline $8024188^{*}$ & 23.849 & 4.384 & 5829 & 23.528 & $1.587 \times 10^{-3}$ & 22.754 & $1.653 \times 10^{-3}$ & 0.0334 & $1.007 \times 10^{-4}$ & 0.00908 \\
\hline $8037792^{* \dagger}$ & 23.400 & 4.285 & 5666 & 24.797 & $3.245 \times 10^{-3}$ & 23.132 & $2.280 \times 10^{-3}$ & 0.0695 & $1.712 \times 10^{-4}$ & 0.01823 \\
\hline $8495770^{* \dagger}$ & 25.634 & 4.545 & 5688 & 26.244 & $2.802 \times 10^{-3}$ & 25.832 & $1.605 \times 10^{-3}$ & 0.0158 & $1.250 \times 10^{-4}$ & 0.00383 \\
\hline 9996105 & 28.683 & 3.990 & 5815 & 30.006 & $1.936 \times 10^{-2}$ & 26.012 & $1.881 \times 10^{-2}$ & 0.1426 & $1.032 \times 10^{-3}$ & 0.03215 \\
\hline $10079452^{*}$ & 26.261 & 4.030 & 5812 & 25.092 & $7.177 \times 10^{-3}$ & 25.842 & $3.767 \times 10^{-3}$ & 0.0294 & $3.230 \times 10^{-4}$ & 0.00726 \\
\hline 10279927 & 24.018 & 4.508 & 5638 & 23.429 & $7.529 \times 10^{-4}$ & 22.373 & $8.778 \times 10^{-4}$ & 0.0461 & $5.166 \times 10^{-5}$ & 0.01265 \\
\hline
\end{tabular}

*Stars with manifestation of DR, which are in common with Reinhold \& Gizon (2015).

${ }^{\dagger}$ Stars with ACFs lower than the threshold 0.6-0.7. 

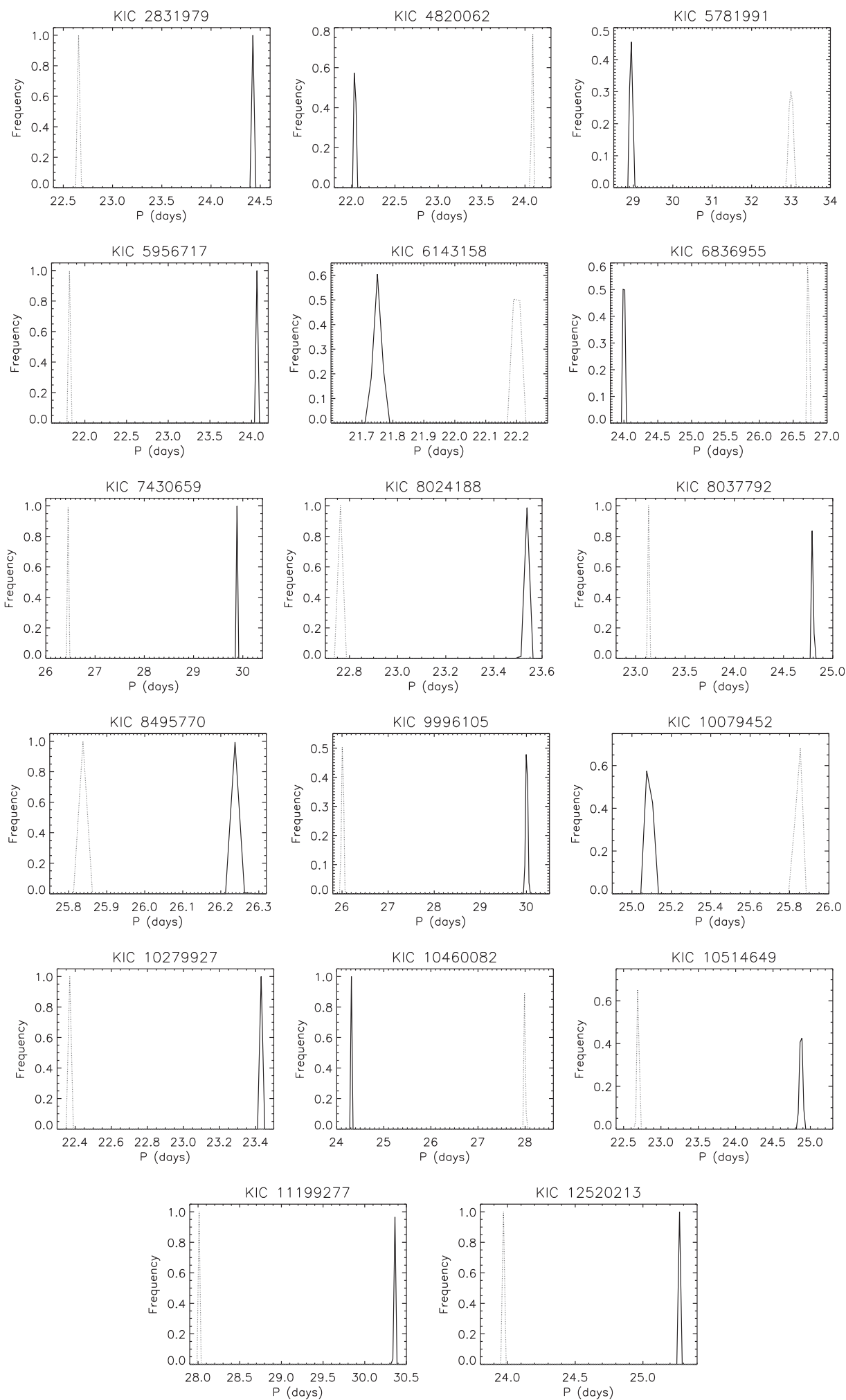

Figure 2. A posteriori distributions of the rotation periods of the two spots as derived from MCMC for all stars. The solid line refers to the distribution of the rotation period of the first spot, and the dashed line refers to that of the second.

lines display the initial and final times $t_{1}$ and $t_{2}$ of the intervals considered for the MCMC analysis. We then applied the procedure by Lanza et al. (2014) to compute the spot rotation period $P$, the mean values of the individual spot rotation periods $P_{1}$ and $P_{2}$ and their respective colatitudes, $\theta_{1}$ and $\theta_{2}$, and the relative ampli- tude of the DR, $\Delta P / P$, where $P=\left(P_{1}+P_{2}\right) / 2$. The a posteriori distributions of the rotation periods $P_{1}$ and $P_{2}$ of the two spots for all 17 stars, as derived from MCMC, are given in Fig. 2. The standard deviations of $\triangle P / P$ were also estimated by a model that assumes that starspots are not evolving along the fitted interval. Starspot 


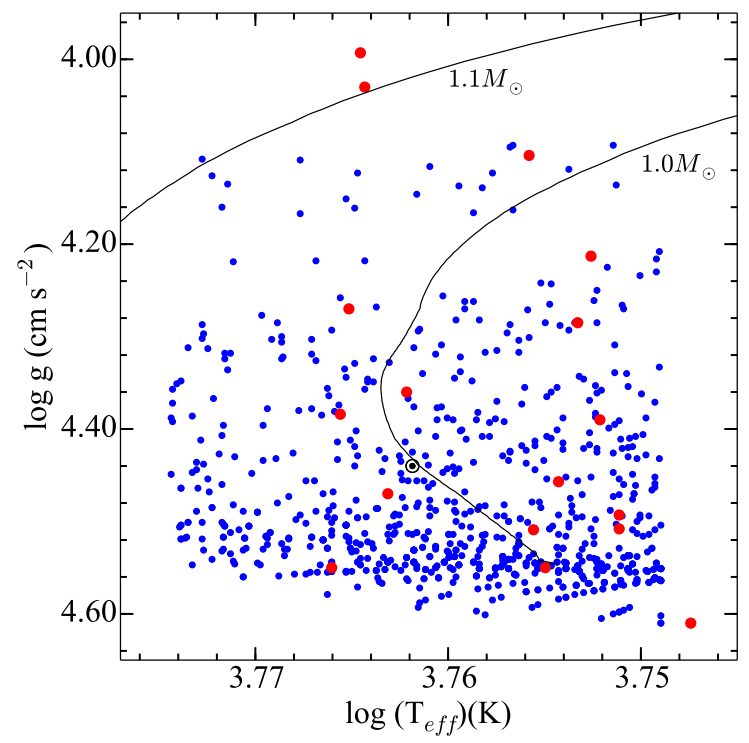

Figure 3. Distribution of Sun-like stars with measured DR in the $\log g$ and $T_{\text {eff }}$ diagram, represented by red circles. Blue circles indicate stars of the original working sample without traces of DR. The evolutionary tracks are from Ekström et al. (2012). The Sun is represented by it usual symbol.

evolution can limit our accuracy in measuring DR at $\Delta \Omega \sim 1 / t_{\mathrm{evol}}$, where $t_{\mathrm{evol}}$ is the evolutionary time-scale, or even mimic a DR signal in the worst cases (see Aigrain et al. 2015).

\section{RESULTS}

The main results of the present study are given in Table 2, which lists the mean values of the individual spot rotation periods $P_{1}$ and $P_{2}$, the relative amplitude of the DR lower limit, $\Delta P / P$ and the amplitude of the DR expressed as the frequency difference between the spots frequencies, $\Delta \Omega$. Table 2 lists also the stellar parameters $P_{\text {rot }}, \log g$ and $T_{\text {eff. }}$. Fig. 3 displays, in the $\log g$ versus $T_{\text {eff }}$ diagram, the locations of the 881 stars defined in our selection criteria, namely stars showing physical properties that are approximately equal to the Sun values, with $3.94 \mathrm{~cm} \mathrm{~s}^{-2}<\log g<4.94 \mathrm{~cm} \mathrm{~s}^{-2}, 5579 \mathrm{~K}$ $<T_{\text {eff }}<5979 \mathrm{~K}$, and the rotation period ranging into the solar values, from $23 \mathrm{~d}<P_{\text {rot }}<33 \mathrm{~d}$. In the referred figure, the red points represent the 17 stars having spot lifetimes long enough for the detection of DR patterns on the basis of our spot-modelling method. Evolutionary tracks taken from Ekström et al. (2012) are overlayed to constrain the view of the mass range and evolutionary stage of the sample stars, with the position of the Sun indicated by the black symbol.

We compared the present results with Reinhold \& Gizon (2015). Indeed, from our sample of 17 stars with measured amplitude of surface DR, 11 stars are found to be in common with those authors. For these common stars, Reinhold \& Gizon (2015) detected the presence of multiple periods in their LCs, which were interpreted as the manifestation of DR, using a different approach based on the Lomb-Scargle periodogram. These stars are identified in Table 2 with an asterisk. A simple comparison between the DR values of this small sample of targets in common provides no correlation between the values, although they are distributed in a similar range. As a more robust test, we applied Student's $t$-test, which can be used to compare whether measures in one sample are paired with measures in another sample. According to this method, the null hypothesis assumes that the true mean difference between the two observations

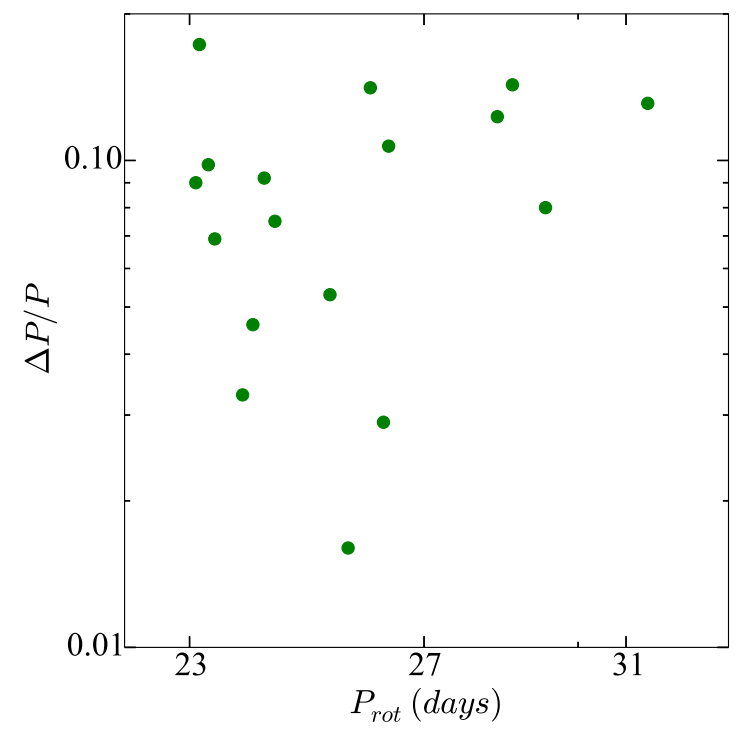

Figure 4. The distribution of the relative amplitude $\Delta P / P$ versus the rotation period $P_{\text {rot }}$ for the 17 Sun-like stars with DR traces identified in the present study.

on each sample is zero; otherwise, the alternative hypothesis is considered. In this sense, the results of the paired $t$-test show that, because the $-t_{0.025}(-2.228)<t_{\text {computed }}(-0.959)<t_{0.025}(2.228)$ and because the $p$-value $>0.05$ (confidence level), we cannot reject the null hypothesis. Such a fact may reflect, in principle, the difference in the nature of the procedures applied in the search for DR traces. In addition, the compatibility between their ranges suggests that their information is valid at least up to an order of magnitude.

Finally, we analysed the behaviour of the relative amplitude $\Delta P / P$ as a function of rotation period for our sample of 17 stars despite the narrow range of rotation periods considered in this study, namely, from 23 to 33 d. Fig. 4 displays the behaviour of $P_{\text {rot }}$ versus $\Delta P / P$, from which one observes a soft trend of increasing $\Delta P / P$ towards longer rotation periods, paralleling the scenario found by different studies. For instance, as shown by Reinhold et al. (2013), the relative DR shear increases with longer rotation periods, in agreement with previous observations (Barnes et al. 2005) and theoretical approaches (Küker \& Rüdiger 2011b).

\section{CONCLUSIONS}

Based on a simple two-spot model together with a BIC, we measured a lower limit on the amplitude of surface DR for $17 \mathrm{Ke}$ pler Sun-like stars. For these stars, using Kepler high-precision and evenly sampled photometric time series, it was possible to compute the spot rotation period $P$, the mean values of the individual spot rotation periods $P_{1}$ and $P_{2}$ and the relative amplitude of the DR, $\Delta P / P$, where $P=\left(P_{1}+P_{2}\right) / 2$. These stars present a soft trend of the estimated relative amplitude, $\Delta P / P$, increasing with increasing rotation periods, in agreement with the scenarios found in the literature, from several observational studies of DR, based on different measurement approaches.

In summary, although the art of measurements of the surface rotation of stars has now been mastered, with a high level of precision and maturity, the detection and measurement of stellar DR remains a tricky subject. In the present study, using a spotmodelling procedure, we were able to detect surface DR patterns in 17 stars with physical properties, including rotation, similar to the 
Sun. The portrait emerging from this study points to a significant perspective: among Sun-like stars with surface rotation similar to the solar values, surface DR appears to be a common phenomenon.

\section{ACKNOWLEDGEMENTS}

The research activity of the Observational Astronomy Board of the Federal University of Rio Grande do Norte (UFRN) is supported by continuous grants from CNPq and FAPERN Brazilian agencies. We also acknowledge financial support from INCT INEspaço/CNPq/MCT. MLC, JPB and ADC acknowledge CAPES/PNPD fellowships. ICL and CEFL acknowledge CNPq/PDE fellowships. RSB and FPC acknowledge graduate fellowships from CAPES. DBdeF also acknowledges financial support by the Brazilian agency CNPq (Grant No. 306007/2015-0). This paper includes data collected by the Kepler mission. Funding for the Kepler mission is provided by the NASA Science Mission Directorate. All Kepler data presented in this paper were obtained from the Mikulski Archive for Space Telescopes (MAST). We would like to thank the anonymous referee for the helpful comments that lead us to a substantial improvement of this manuscript.

\section{REFERENCES}

Aerts C. et al., 2010, A\&A, 513, L11

Affer L., Micela G., Favata F., Flaccomio E., 2012, MNRAS, 424, 11

Aigrain S. et al., 2015, MNRAS, 450, 3211

Baglin A., Auvergne M., Barge P., Deleuil M., Catala C., Michel E., Weiss W., COROT Team, 2006, in Fridlund M., Baglin A., Lochard J., Conroy L., eds, ESA Special Publication Vol. 1306, Scientific Objectives for a Minisat: CoRoT. ESA, Noordwijk, p. 33

Baliunas S. L. et al., 1983, ApJ, 275, 752

Barnes J. R., Collier Cameron A., Donati J.-F., James D. J., Marsden S. C., Petit P., 2005, MNRAS, 357, L1

Basri G. et al., 2011, AJ, 141, 20

Bonomo A. S., Lanza A. F., 2012, A\&A, 547, A37

Borucki W. J. et al., 2010, Science, 327, 977

Bouvier J., Forestini M., Allain S., 1997, A\&A, 326, 1023

Browning M. K., 2008, ApJ, 676, 1262

Brun A. S., 2004, in Danesy D., ed., ESA Special Publication Vol. 559, SOHO 14 Helio- and Asteroseismology: Towards a Golden Future. ESA, Noordwijk, p. 271

Collier Cameron A., Donati J.-F., Semel M., 2002, MNRAS, 330, 699

Croll B. et al., 2006, ApJ, 648, 607

De Freitas D. B., Leão I. C., Ferreira Lopes C. E., Paz-Chinchon F., Canto Martins B. L., Alves S., De Medeiros J. R., Catelan M., 2013, ApJ, 773, L18

De Medeiros J. R., Udry S., 1999, A\&A, 346, 532

De Medeiros J. R. et al., 2013, A\&A, 555, A63

De Medeiros J. R., Alves S., Udry S., Andersen J., Nordström B., Mayor M., 2014, A\&A, 561, A126

Davenport J. R. A., Hebb L., Hawley S. L., 2015, ApJ, 806, 212
Eggenberger P. et al., 2010, A\&A, 519, A116

Ekström S. et al., 2012, A\&A, 537, A146

Fröhlich H.-E., 2007, Astron. Nachr., 328, 1037

Fröhlich H.-E., Frasca A., Catanzaro G., Bonanno A., Corsaro E., MolendaZakowicz J., Klutsch A., Montes D., 2012, A\&A, 543, A146

Gizon L., Solanki S. K., 2004, Sol. Phys., 220, 169

Huber D. et al., 2014, ApJS, 211, 2

S. V., Keller C. U., 2009, in Stempels E., ed., AIP Conf. Proc. Vol. 1094, Cool Stars, Stellar Systems and the Sun. Am. Inst. Phys., New York, p. 664

Jenkins J. M. et al., 2010, ApJ, 713, L87

Käpylä P. J., Mantere M. J., Brandenburg A., 2012, ApJ, 755, L22

Kawaler S. D., 1988, ApJ, 333, 236

Kitchatinov L. L., 2013, in Kosovichev A. G., de Gouveia Dal Pino E., Yan Y., van Driel -Gesztelyi L., eds, Proc. IAU Symp. 294, Solar and Astrophysical Dynamos and Magnetic Activity. Cambridge Univ. Press, Cambridge, p. 399

Kitchatinov L. L., Olemskoy S. V., 2011, MNRAS, 411, 1059

Kovári Z. et al., 2015, A\&A, 573, A98

Küker M., Rüdiger G., 2005, Astron. Nachr., 326, 265

Küker M., Rüdiger G., 2011a, Astron. Nachr., 332, 83

Küker M., Rüdiger G., 2011b, Astron. Nachr., 332, 933

Lanza A. F., Rodono M., Zappala R. A., 1993, A\&A, 269, 351

Lanza A. F., Das Chagas M. L., De Medeiros J. R., 2014, A\&A, 564, A50

Leão I. C. et al., 2015, A\&A, 582, A85

Lebedinsky A. I., 1941, Astron. Zh, 18, 10

McQuillan A., Aigrain S., Mazeh T., 2013, MNRAS, 432, 1203

McQuillan A., Mazeh T., Aigrain S., 2014, ApJS, 211, 24

Miesch M. S., 2005, Living Rev. Sol. Phys., 2, 1

Miesch M. S., Toomre J., 2009, Annu. Rev. Fluid Mech., 41, 317

Nordström B. et al., 2004, A\&A, 418, 989

Oláh K., Jurcsik J., Strassmeier K. G., 2003, A\&A, 410, 685

Reiners A., Mohanty S., 2012, ApJ, 746, 43

Reiners A., Schmitt J. H. M. M., 2003, A\&A, 398, 647

Reinhold T., Gizon L., 2015, A\&A, 583, A65

Reinhold T., Reiners A., 2013, A\&A, 557, A11

Reinhold T., Reiners A., Basri G., 2013, A\&A, 560, A4

Shu F., Najita J., Ostriker E., Wilkin F., Ruden S., Lizano S., 1994, ApJ, 429, 781

Smith J. C. et al., 2012, PASP, 124, 1000

Strassmeier K. G., Kratzwald L., Weber M., 2003, A\&A, 408, 1103

Stumpe M. C. et al., 2012, PASP, 124, 985

Thompson M. A. et al., 2013, PASP, 125, 809

Twicken J. D., Chandrasekaran H., Jenkins J. M., Gunter J. P., Girouard F., Klaus T. C., 2010, in Radziwill N. M., Bridger A., eds, Proc. SPIE Conf. Ser. Vol. 7740, Software and Cyberinfrastructure for Astronomy. SPIE, Bellingham, p. 1

Van Cleve J. E. et al., 2010, American Astronomical Society Meeting Abstracts. p. 420.02

van Saders J. L., Pinsonneault M. H., 2013, ApJ, 776, 67

Vida K., Kovári Z., Švanda M., Oláh K., Strassmeier K. G., Bartus J., 2007, Astron. Nachr., 328, 1078

Weber M., Strassmeier K. G., Washuettl A., 2005, Astron. Nachr., 326, 287 


\section{APPENDIX：FIGURES}
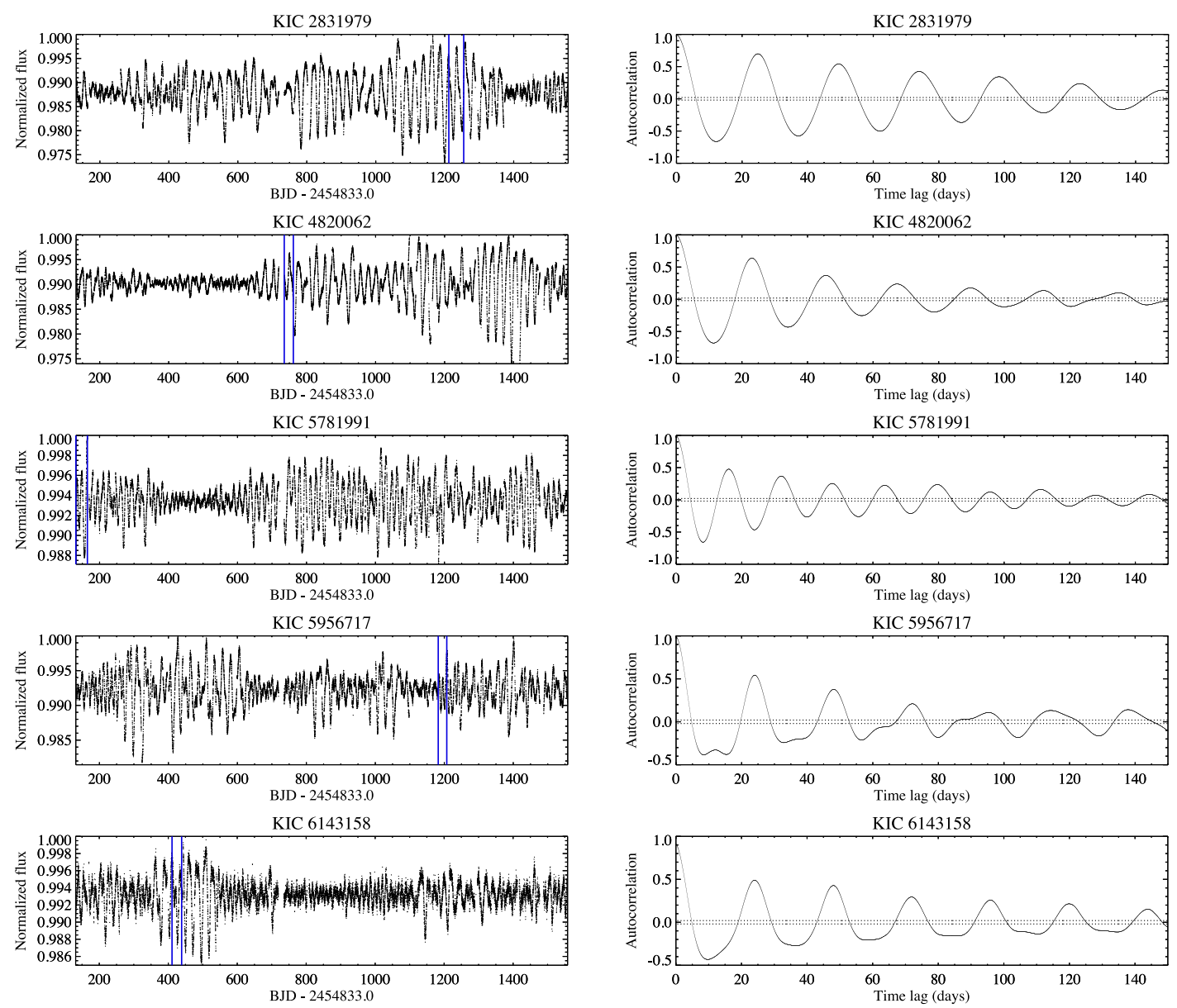

KIC 6836955
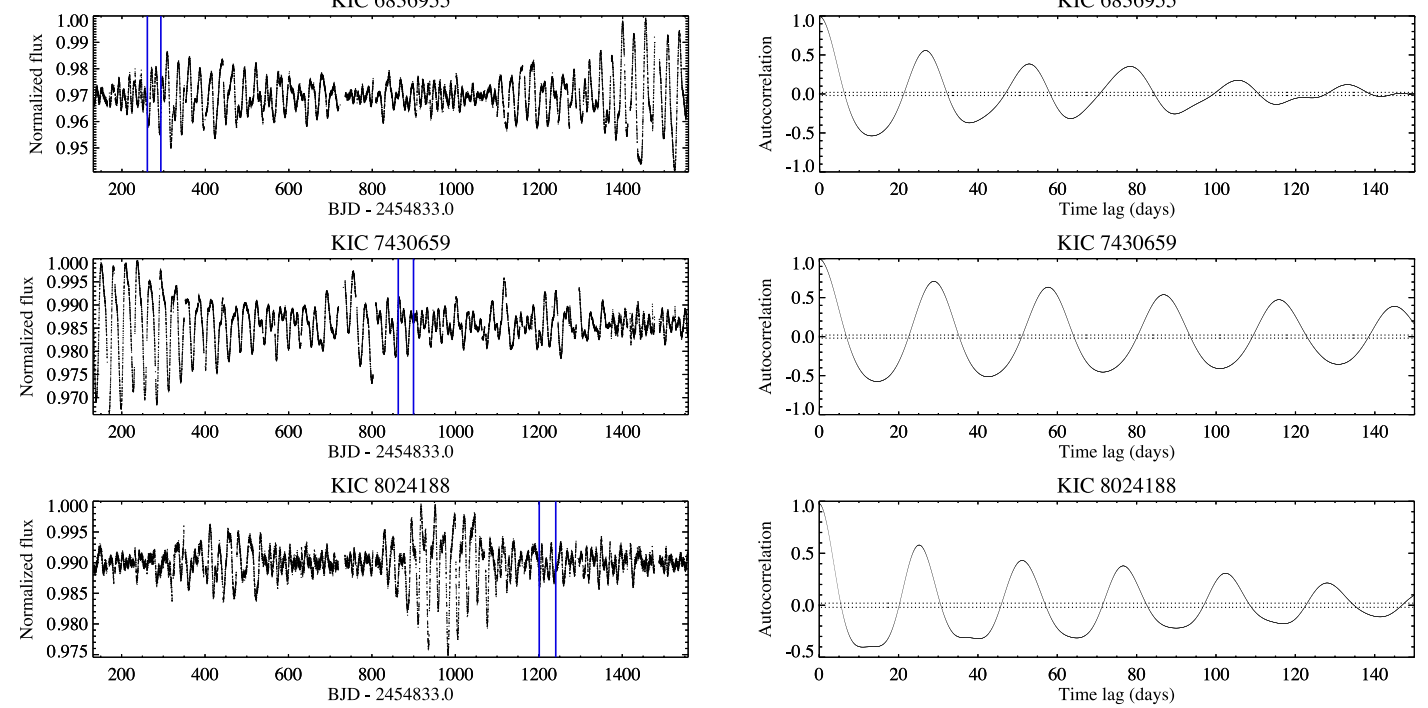

Figure A1. Left: photometric times series of Kepler stars (from top to bottom) KIC 2831979, KIC 4820062, KIC 5781991, KIC 5956717, KIC 6143158, KIC 6836955, KIC 7430659, KIC 8024188, KIC 8037792, KIC 8495770, KIC 9996105, KIC 10079452, KIC 10279927, KIC 10460082, KIC 10514649, KIC 11199277 and KIC 12520213. The flux has been normalized to the maximum value observed along each time series. The vertical solid lines (in blue) display the initial and final times of the intervals considered for MCMC analysis (see Table 1). Right: autocorrelation functions of the LCs of the stars in our sample. The dotted lines indicate the interval corresponding to $\pm \sigma$, where $\sigma$ is one standard deviation of the autocorrelation as expected for a pure random noise with some degree of autocorrelation according to the large-lag approximation. 

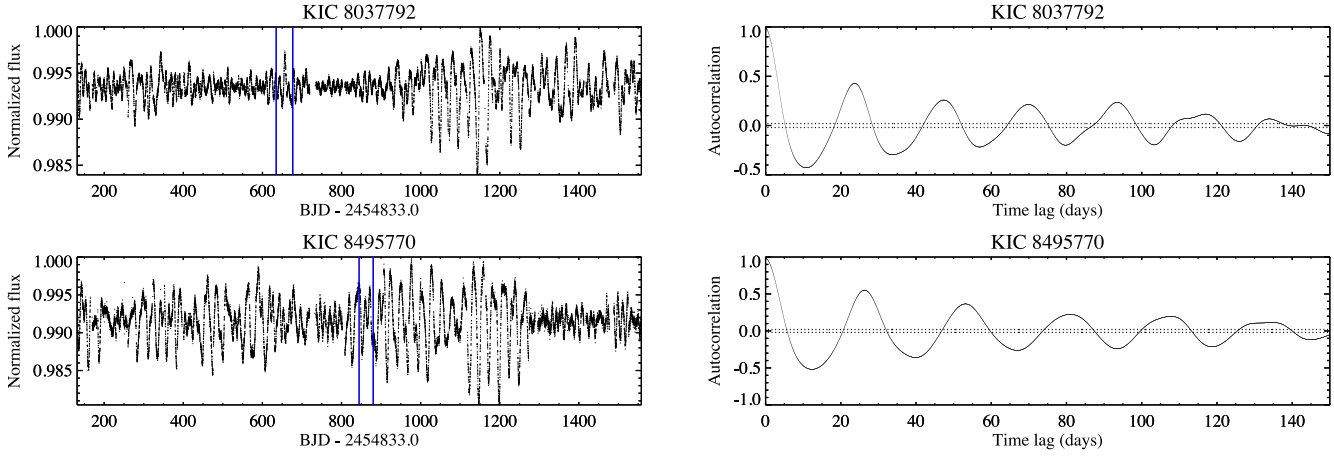

KIC 8495770
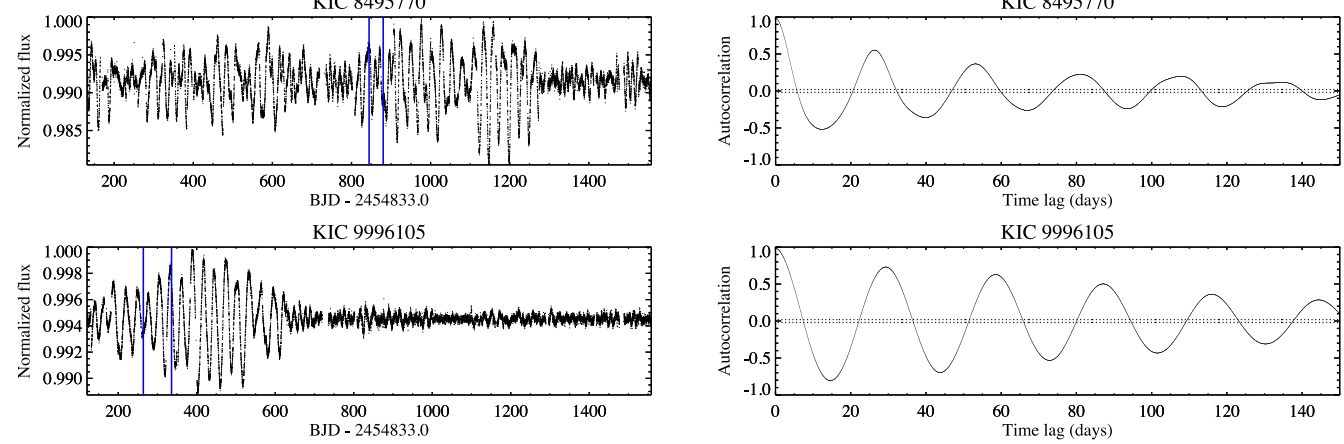

KIC 9996105

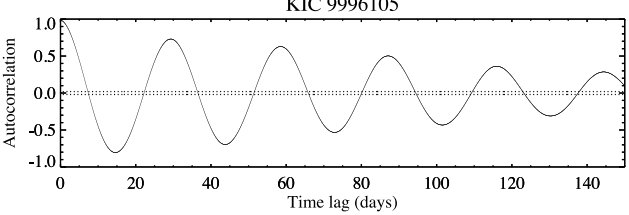

KIC 10079452

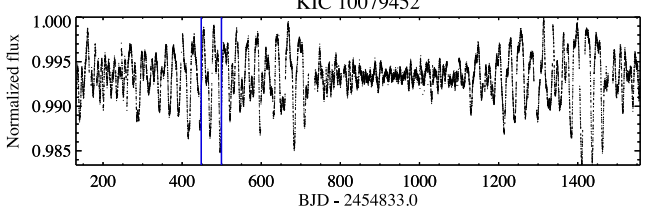

KIC 10079452

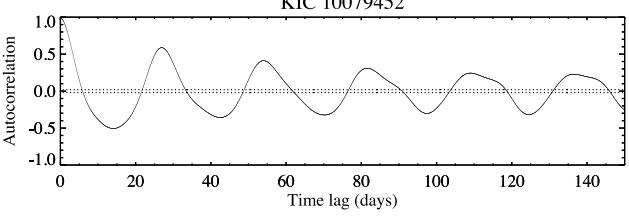

KIC 10279927

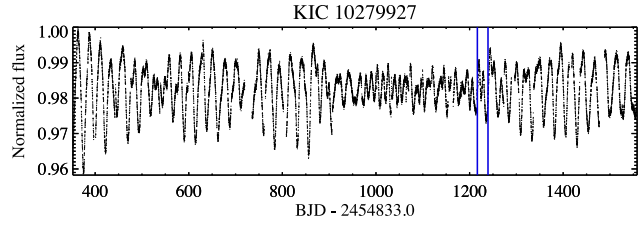

KIC 10279927

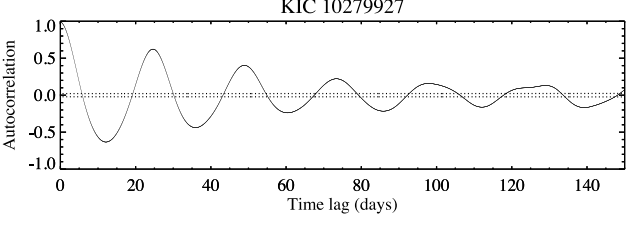

KIC 10460082

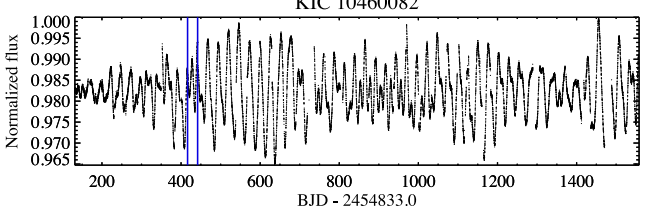

KIC 10460082
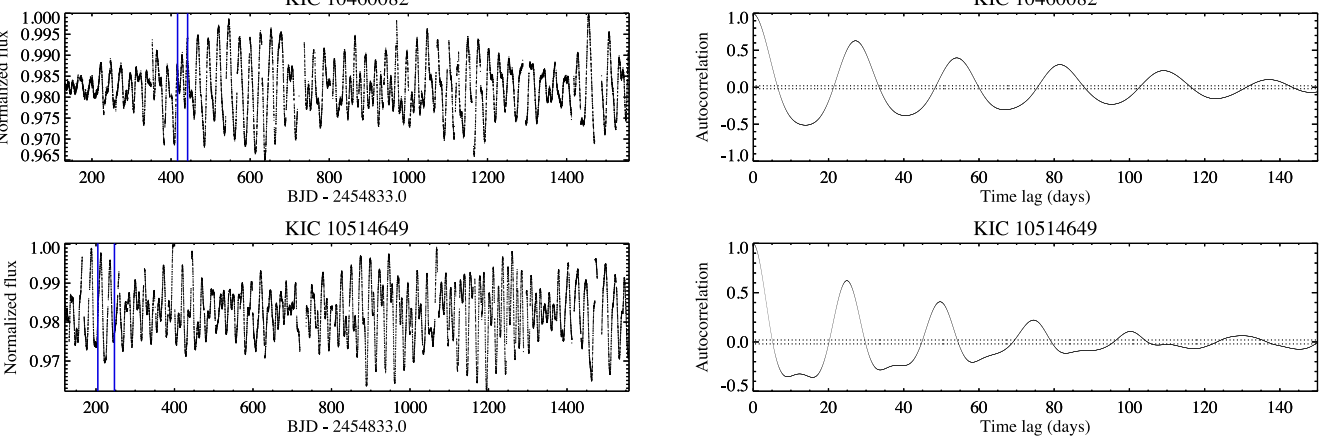

KIC 11199277

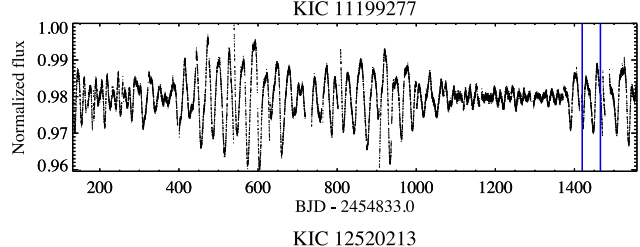

KIC 11199277

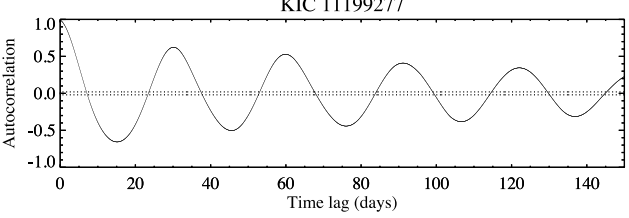

KIC 12520213
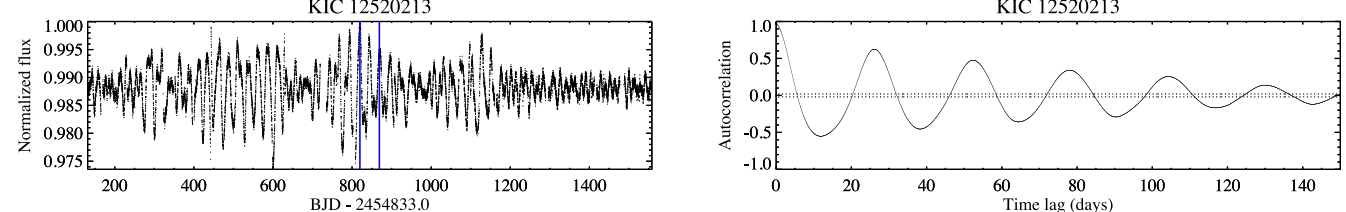

Figure A1 - continued

This paper has been typeset from a $\mathrm{T}_{\mathrm{E}} \mathrm{X} / \mathrm{LAT} \mathrm{EX}$ file prepared by the author. 